山्山FFRANÇAISE

$\supset \mathrm{DE}$

$\stackrel{1=1}{\simeq}$ PÉDAGOGIE

\section{Revue française de pédagogie}

Recherches en éducation

159 | avril-juin 2007

Politique et rhétoriques de l'« École juste » avant la Cinquième République

\title{
Figures de l'école juste et politique scolaire dans les années fondatrices de la Troisième République
}

The features of fair schooling and school policies in the founding years of the Third Republic

Figuras de la escuela justa y política escolar en los años fundadores de la Tercera República

Gestalten der gerechten Schule und Schulpolitik in den Gründerjahren der 3.

Republik

\section{Patrick Dubois}

\section{OpenEdition}

Journals

Édition électronique

URL : http://journals.openedition.org/rfp/646

DOI : $10.4000 /$ rfp. 646

ISSN : 2105-2913

Éditeur

ENS Éditions

Édition imprimée

Date de publication : 1 avril 2007

Pagination : 13-22

ISBN : 978-2-7342-1090-0

ISSN : 0556-7807

Référence électronique

Patrick Dubois, «Figures de l'école juste et politique scolaire dans les années fondatrices de la

Troisième République », Revue française de pédagogie [En ligne], 159 | avril-juin 2007, mis en ligne le 01 avril 2011, consulté le 23 avril 2019. URL : http://journals.openedition.org/rfp/646 ; DOI : 10.4000/ rfp.646 


\section{Figures de l'école juste et politique scolaire dans les années fondatrices de la Troisième République}

Patrick Dubois

L'article confronte la politique républicaine des ordres séparés aux discours publics de justification ou de dénonciation dont elle a fait l'objet en son temps. Trois figures de contestation de cette politique sont dégagées, conservatrice, intégraliste et réformiste. Dès les années 1880, à travers l'idée d'une école primaire universelle, et au nom du principe méritocratique lui-même dont se réclame l'école républicaine, un rapprochement effectif des publics des deux séries d'établissements primaire et secondaire commence à être pensé, sinon revendiqué.

Descripteurs (TEE) : 1880-1889, école, France, politique de l'éducation, système d'enseignement.

$\mathrm{L}$ 'école de Jules Ferry était-elle juste? II est convenu aujourd'hui de souligner que les grandes lois scolaires des années 1880 n'ont jamais favorisé cette "première fusion qui résulte du mélange des riches et des pauvres sur les bancs de quelque école ", appelée de ses vœux en avril 1870 par le jeune député de Paris, J. Ferry, dans son discours " sur l'égalité » à la salle Molière (Ferry, 1893-1898, t. 1, p. 283-305), mais qu'elles ont au contraire préservé la forte partition scolaire et sociale des ordres d'enseignement: d'un côté, le primaire et le primaire supérieur; de l'autre, l'enseignement secondaire, commencé par ses classes élémentaires et leur professorat spécifique, institué par Jules Ferry lui-même. II n'est pas sûr, toutefois, que l'idée sensiblement postérieure d'égalité des chances, à partir de laquelle ce jugement est porté - et parfois ce procès instruit - soit à elle seule pertinente pour ressaisir les enjeux sociaux de la politique scolaire républicaine de la période. Pour comprendre comment et jusqu'à quel point ce qui a été réprouvé ultérieurement comme une discrimination sociale a pu être jugé par les contemporains compatible avec les principes républicains de justice et d'égalité, il nous a paru plus fécond de confronter cette politique scolaire républicaine des ordres à l'espace des positions ouvert par les discours publics de justification et de dénonciation dont elle a fait l'objet en son temps. 


\section{LE PARADIGME MÉRITOCRATIQUE DANS LA JUSTIFICATION RÉPUBLICAINE DES ORDRES}

Dans un long discours à la Chambre le 6 juin 1889, Jules Ferry (1893-1898, t. 4, p. 430-480) revient sur les lois scolaires des années 1880, dont il a été le principal artisan. Citant approximativement Condorcet - «l'enseignement est un devoir de justice envers les citoyens »-, il donne à ce devoir une signification que le philosophe des Lumières n'aurait pas désavouée : "la société doit à tous le nécessaire du savoir pratique, et l'avènement aux degrés successifs de la culture intellectuelle de tous ceux qui sont aptes à les franchir ». Dérivé de la Déclaration des droits de l'homme et légitimé par l'héritage révolutionnaire, le paradigme méritocratique qui s'exprime ici est un des lieux communs de la période: on s'accorde en son nom sur la nécessité d'un premier degré d'instruction pour tous et l'on réserve aux plus aptes ou aux plus méritants les degrés les plus élevés. II est notamment formulé chaque fois que la pulsion patriotique conduit un acteur républicain à revendiquer pour le profit de la nation une politique volontariste de promotion vers l'enseignement secondaire des meilleurs éléments du primaire. C'est, par exemple, le recteur gambettiste Pierre Foncin qui, dès 1879, à la cérémonie d'ouverture des facultés de Douai, réclamait à cette fin, sur un mode plus incantatoire que pragmatique, l'abolition des "barrières vermoulues " entre les ordres: "La société absolue des trois ordres primaire, secondaire et supérieur était bonne au temps où la société elle-même se croyait divisée en classes ennemies; abattons ces barrières vermoulues. Soyons comme la nation elle-même, qui est une et indivisible... L'intérêt suprême de la patrie exige qu'il n'y ait aucune déperdition de forces dans l'ensemble de la nation. L'instituteur n'est donc pas seulement chargé de donner l'enseignement primaire; il lui appartient encore d'observer ses élèves avec le plus grand soin, d'étudier, d'éveiller leurs facultés naissantes, de trier parmi les mieux doués ceux qui pourront occuper utilement une place dans l'enseignement secondaire » (1).

Cette utopie méritocratique d'un espace scolaire hiérarchisé, où les positions acquises ne résulteraient que du talent individuel, pouvait d'ailleurs à bon droit constituer l'horizon réaliste d'une politique scolaire juste. Dès les années 1870, en effet, des rapports officiels avaient convaincu une fraction des élites républicaines - J. Ferry et F. Buisson sont du nombre - des progrès accomplis ailleurs dans cette direction, principalement aux États-Unis (Hippeau, 1870 ; Buisson, 1878).
Dans le débat public de la période, toutefois, un autre principe d'organisation scolaire surplombe l'idée méritocratique, qui appelle à une appropriation nécessaire des structures d'enseignement aux clientèles sociales. Exprimée ou sous-entendue, cette conviction qui installe la diffusion des Lumières dans un système articulé des besoins sociaux est alors partagée "par à peu près tous ceux qui s'intéressent aux questions d'enseignement, universitaires ou publicistes » (2). Elle perpétue en quelque manière, tout en la réinscrivant dans le contexte du suffrage universel, qui impose la mise en œuvre d'une acculturation primaire de tous les citoyens, l'idéologie doctrinaire de la Monarchie de Juillet (Rosanvallon, 1985). C'est ainsi que, dans le souci de consolider des filières d'enseignement à destination de la «partie des classes moyennes qui ne saurait se contenter de l'instruction primaire et à qui les études classiques ne sauraient convenir » (Marguerin, 1878, p. 345), l'administration républicaine a poursuivi les tentatives amorcées par les régimes précédents, à la fois de développement de l'enseignement secondaire spécial (3), en plein essor dans les années 1880 (Gréard, 1887, vol. 3, p. 121, note) ; et d'extension de l'enseignement primaire, avec la réorganisation de l'enseignement primaire supérieur et des écoles municipales professionnelles des grandes villes (Chapoulie, 1989 ; Thivend, 2006).

Or cet idéal d'homologie entre la morphologie des clientèles sociales et les structures d'enseignement s'est trouvé au principe d'une double tension, sociale et politique, qui a mobilisé chez les acteurs républicains diverses rhétoriques de justification. La première est liée à la concurrence entre les deux réseaux scolaires intermédiaires, exposant l'enseignement secondaire à l'afflux de publics inappropriés, dont les aspirations pouvaient outrepasser leur destination sociale (Marguerin, 1881, p. 130-131). Même quand il se veut rassurant, le discours officiel laisse transparaître, en creux, l'inquiétude du désordre social que pourrait engendrer un usage perverti du savoir, conduisant des sujets à une revendication indue de modification de leur état. Lorsqu'en 1878 Octave Gréard, alors directeur de l'enseignement primaire de la Seine, examine méticuleusement les résultats de la statistique de l'enseignement primaire supérieur à Paris de 1867 à 1877, il se félicite que les élèves de ces établissements, "que la nature a doués d'aptitudes exceptionnelles », cherchent par ce type d'enseignement «à s'élever dans leur sphère, mais non à en sortir ». Ainsi confinée dans la «sphère » des petites classes moyennes laborieuses d'où ils sont issus, leur aspiration individuelle demeure " conforme 
à l'intérêt public » (Gréard, 1887, vol. 1, p. 309). Lecteur du rapport d'Octave Gréard, Félix Pécaut, alors publiciste au Temps, se réjouit lui aussi que «le péril du déclassement social n'est pas aggravé par l'extension ni surtout par le perfectionnement de l'instruction populaire " et qu'il en est " plutôt diminué " (Pécaut, 1879, p. 179). Jules Ferry lui-même, dans son rapport de 1881 au Président de la République sur l'enseignement primaire supérieur, évoque les inquiétudes de quelques "bons esprits " qui redoutaient, à la création de classes supérieures du primaire, que la "vanité des familles " ou «l'amour propre des municipalités " n'en fasse " une contrefaçon malheureuse de l'enseignement secondaire spécial » (Ferry, 1881b, p. 675-676) ; et il ajoute, sous la forme d'un constat qui est aussi une injonction, que le « bon sens de notre pays " a empêché la transformation de ces écoles en "collèges dégénérés ", rappelant qu'elles se devaient d'acheminer leurs élèves, " non pas théoriquement vers toutes les professions, mais positivement vers celles auxquelles les prédestine le milieu natal » (Ibid.).

L'autre tension se situe sur le plan proprement politique : la réalité d'une politique scolaire socialement différenciatrice met à l'épreuve l'idée d'égalité des droits. Pour les républicains de gouvernement, le principe démocratique, autant que le besoin d'unité nationale imposaient, au-delà de la mise en œuvre d'une acculturation primaire de tous les citoyens, des mesures de rapprochement des ordres scolaires. Le "point de vue démocratique ", expliquait ainsi Jules Ferry dans un discours à la Chambre le 13 juillet 1880, " c'est qu'il importe à une société comme la nôtre, à la France d'aujourd'hui, de mêler sur les bancs de l'école les enfants qui se trouveront, un peu plus tard, mêlés sous le drapeau de la patrie " (Ferry, 1893-1898, t. 4, p. 39). Dans cet esprit, à défaut d'une instruction primaire commune fondée sur une réelle mixité sociale (4), quelques mesures ont été prises, on le sait, pour assouplir la frontière entre les ordres, qui ont au moins facilité la rhétorique de leur unité symbolique, et même de leur enchaînement possible. Certes, dès la première réunion de la section permanente du Conseil supérieur de l'Instruction publique, en mai 1880, Jules Ferry avait proposé un nouveau plan d'études pour l'enseignement secondaire, qui le divisait en trois cycles séparés l'un de l'autre par des examens de passage, ce qui devait permettre à d'excellents sujets de milieu modeste d'entrer dans un établissement secondaire classique sans être contraint d'en parcourir tout le curriculum jusqu'au baccalauréat (Archives Nationales, $\mathrm{F} / 17 / 3212)$; mais devant les objections présentées à ce plan par les défenseurs de l'enseignement secondaire classique, le ministre avait reculé. Tout au plus, dans le plan d'études du 2 août 1880, la première période - les classes élémentaires, désormais sans latin - avait été maintenue "dans les limites d'un bon enseignement primaire " (Boissier, 1880, p. 107). Complémentairement, Jules Ferry s'était employé à compenser symboliquement le clivage social entre les ordres en transférant dans les missions de l'école primaire des finalités et valeurs jusque là réservées, comme sa marque distinctive, à l'ordre secondaire (et même à l'ordre secondaire classique). Le syntagme d'« éducation libérale ", utilisé par lui une première fois en avril 1881, devant une délégation d'instituteurs réunis en congrès à la Sorbonne, pour caractériser l'enseignement primaire républicain, encore appelé le même jour "enseignement secondaire au petit pied (Ferry, 1881a, p. 580), octroyait ainsi au curriculum primaire une finalité éducative, à l'égal du secondaire.

Jointes à ce qu'il nomme le "bienfait incessamment accru des bourses d'État ", ces quelques dispositions prises pour réduire le fossé entre les ordres conduisent J. Ferry, dès 1881, à écrire que l'enseignement secondaire se trouve désormais "directement rattaché à l'enseignement élémentaire " et qu'une "large issue " au premier est dorénavant ouverte au second. Par cette issue pourtant modeste - en 1883, 2622 lycéens sur un total de 48313 sont boursiers (Buisson, 1878-1887, $1^{\text {re }}$ partie, t. 2, p. 1751-1752) -, des acteurs républicains, tel l'ancien pasteur protestant libéral et député Jules Steeg, voient l'aménagement d'un passage " de plain-pied » entre l'école communale et le lycée : " désormais un élève sortant de l'école primaire communale peut entrer de plainpied dans la classe de quatrième du lycée, sans qu'il existe aucune différence de préparation entre lui et ceux de ses camarades qui ont suivi les classes élémentaires d'un établissement secondaire " (article "Lycées et collèges » apud Buisson, 1878-1887, $1^{\text {re }}$ partie, t. 2, p. 1748 B).

\section{ORDRES SÉPARÉS ET PRINCIPE DÉMOCRATIQUE : L'ÉLITISME RÉPUBLICAIN EN QUESTION}

Si la question de la relation entre les ordres d'enseignement n'a pas déchaîné dans les années 1880 des passions comparables à celles qui ont accompagné le vote des lois scolaires relatives à l'enseignement primaire, des formes diverses de contestation se sont 
pourtant fait entendre à son sujet, au sein même du camp républicain, parfois portées par des personnalités bénéficiant d'une notoriété, et donc d'une audience, sur la scène littéraire ou politique, parfois confinées au contraire dans des cercles marginaux et vite désamorcées par la critique de commentateurs autorisés. On peut en distinguer trois figures - conservatrice, intégraliste ou réformiste - qui, chacune en référence à son utopie d'un monde scolaire juste, mettent à l'épreuve l'élitisme scolaire mis en place par les républicains au pouvoir.

\section{La hantise conservatrice d'un passage « de plain-pied » du primaire au secondaire}

Chez les républicains eux-mêmes, l'idée d'une remise en cause du cloisonnement des ordres scolaires a suscité l'émoi d'un certain nombre de personnalités. Formulée radicalement, leur protestation cherche à dénouer la tension entre la séparation sociale des ordres et la revendication démocratique par la disqualification de cette dernière, qui n'est plus alors qu'un "rigorisme démocratique » poussé «jusqu'à s'indigner quand on prononce les noms de classes ou de couches sociales »(Beaussire, 1879, p. 767), voire « une passion niveleuse ", dont « les publicistes les plus éminents ont tant de fois montré les illusions et les abus " (Joly, 1885, p. 280) ; sous une variante plus modérée, elle ne remet pas en cause le principe méritocratique d'égal accès des meilleurs aux degrés les plus élevés de l'instruction, mais considère que l'entrée au collège ou au lycée, chaque année, de quelques excellents éléments du primaire, grâce aux bourses, suffit à sa mise en œuvre. Dans les deux cas, elle récuse comme "un rêve chimérique » l'idée d'un passage «de plain-pied » du primaire au secondaire (Ibid., p. 288 et 303 ) et rejette toute modification du curriculum classique, idéalement adapté à la formation d'une élite dont l'ensemble du corps social pourra tirer profit. Relayée par des périodiques d'opinion, comme la Revue des deux mondes, ou par des publicistes pédagogiques attachés à la défense d'un enseignement classique d'où ils sont issus, cette dénonciation trouve son aliment dans la longue plainte des professionnels de l'enseignement secondaire classique contre le plan d'études de 1880.

Au sein de la Société d'étude des questions d'enseignement secondaire, en particulier, la question de l'éviction du latin des classes élémentaires des lycées est un débat récurrent. Ainsi, lors de la séance du 7 janvier 1885, qui réunit le groupe de l'enseignement classique de la Société, la majorité des présents dénonce une fois de plus la réforme, inspirée par nul motif pédagogique, mais seulement et fâcheusement par «une pensée politique »: favoriser l'accès de l'enseignement secondaire - « nos » lycées! - aux meilleurs éléments du primaire. Le compte rendu de la discussion révèle un argumentaire composite, mais aimanté par un objectif commun, le retour du latin dans la classe élémentaire de $7^{\mathrm{e}}$ des lycées, sinon dans celle de $8^{e}$ : selon ces professionnels de l'enseignement classique, en effet, les élèves des écoles primaires ne sauront pas davantage l'anglais ou l'allemand, substitués au latin dans les classes élémentaires des lycées, que la langue ancienne; à supposer qu'ils le sachent, grâce à un personnel primaire progressivement formé aux langues étrangères, un nouveau privilège naîtrait en faveur des écoles de ville, où ces langues seraient enseignées, au détriment de celles des campagnes; on a « bouleversé les études classiques " pour satisfaire un nombre infime d'élèves du primaire demandant à suivre l'enseignement secondaire ; au reste, les élèves vraiment doués « et qu'il y aurait intérêt pour la société et pour l'État » à faire entrer dans l'enseignement classique, se feront toujours connaître et rattraperont vite le terrain perdu; mais les élèves du primaire scolarisés en lycée répondent rarement aux espérances, par défaut de cette « souplesse d'esprit » que seul l'enseignement secondaire offre, dès ses classes élémentaires, etc. Argumentaire disparate, sans doute, mais cimenté par la double hantise d'un curriculum classique abîmé par l'esprit réformateur et d'une dénaturation rapide de l'enseignement secondaire par l'afflux d'une clientèle inappropriée (L'Université : journal des questions d'instruction publique, 1885, p. 38-39)

\section{La dénonciation intégraliste du paradigme méritocratique}

Tout au long du $\mathrm{XIX}^{\mathrm{e}}$ siècle, les courants socialistes et communistes avaient élaboré, à partir d'une critique radicale de la société bourgeoise et de ses écoles, reproductrices d'inégalités sociales, l'idée d'un enseignement révolutionnaire, abolissant la séparation des ordres primaire et secondaire, et réconciliant l'individu avec lui-même, grâce au développement de l'intégralité de ses facultés que permettrait l'association étroite entre l'instruction intellectuelle et l'apprentissage manuel. À la fin des années 1860, au sein de l'Association internationale des travailleurs, cette utopie scolaire avait pris le nom d'« instruction intégrale ». Quoiqu'elle se revendique elle aussi de l'héritage de la "grande» Révolution (1789), 
l'« instruction intégrale » ne se reconnaît pas dans le paradigme méritocratique: plutôt que d'organiser la sélection des meilleurs, il s'agit ici, en rejetant la notion d'ordres d'enseignement, d'accorder à chacun un droit égal à exploiter les talents qui sont les siens - d'une dignité équivalente à tous les autres ; autrement dit, d'offrir à tous un curriculum encyclopédique abolissant l'antique ligne de partage entre les tours de main artisanaux ou industriels et les habiletés intellectuelles (Demeulenaere-Douyère, 1994). Portée par le mouvement socialiste, l'« instruction intégrale » est réclamée sous la Commune dans de nombreuses réunions politiques. Elle sent donc le soufre après 1871 . On en retrouve pourtant la revendication, à partir de 1876, dans une revue militante proche de la libre pensée, L'École nouvelle, revue de l'éducation intégrale scientifique, industrielle et artistique, animée par Gustave Francolin. Mais son audience est réduite.

À de rares occasions, toutefois, l'« instruction intégrale " fait surface dans le débat public, mobilisée par l'activisme protestataire radical comme paradigme de substitution au modèle scolaire républicain des années 1880. Lorsque G. Clemenceau, tribun de l'extrême-gauche, interpelle J. Ferry à la Chambre, le $1^{\mathrm{er}}$ février 1884, et critique, en invoquant le « principe fondamental du régime démocratique ", l'insuffisance des réformes scolaires en cours, il la revendique explicitement, pour équiper le futur ouvrier ou paysan du savoir "technique » oublié par les réformes républicaines: "Vous voulez retenir les hommes et les enfants dans les campagnes! Intéressez-les alors aux choses de la campagne; montrez-leur ce que c'est que l'agriculture; faites-leur connaître ce que sont cette pierre que rencontre le soc de la charrue, ce brin d'herbe, ce grain de blé ; donnez-leur l'enseignement technique [...] Vous admettez cet enseignement universel, éducation du corps, de l'intelligence, enseignement scientifique, soudé à l'enseignement technique et le précédant; vous reconnaissez que cette éducation intégrale est due à chaque citoyen. Voilà qui est dit. Mais encore, cet enseignement, fautil le donner... » (JO Chambre des députés, $1^{\mathrm{er}}$ février 1884).

Bien que cette idée d'« éducation intégrale » n'ait jamais représenté une menace pour l'ordre scolaire établi, les républicains modérés ou conservateurs en dénoncent unanimement l'idée, laissant entendre, comme O. Gréard, qu'elle défie le bon sens en supposant l'« égalité absolue » des dispositions naturelles - l'adjectif « absolu », à lui seul, suffisant déjà à son discrédit (Gréard, 1887). De même, le minis- tre Goblet, dans un discours aux élèves de l'École polytechnique en 1885, rappelait que l'école républicaine est aux antipodes de " ce rêve insensé d'obtenir l'égalité absolue des intelligences, des facultés, des situations » (Goblet, 1885, p. 332). Plus crûment, Jules Simon, commentant O. Gréard, repérait dans l'idée d'instruction intégrale une menace pour l'ordre social: «C'est pour le coup qu'il deviendrait difficile d'avoir des domestiques dans sa maison et des soldats dans ses corps de garde » (Simon, 1888, p. 196)

\section{Critique réformiste de l'élitisme social républicain}

En 1887, un auteur peu suspect de sympathie pour les thèses de l'enseignement intégral, Édouard Maneuvrier, remet en cause lui aussi la méritocratie républicaine des années 1880. Ancien élève de l'ENS, reçu premier à l'agrégation de philosophie en 1868 , mais engagé ensuite dans une carrière industrielle, il fait paraître un livre qui a eu une réelle audience dans les milieux réformateurs de l'Instruction publique, L'éducation de la bourgeoisie sous la République (Maneuvrier, 1888). L'ouvrage ambitionne d'œuvrer à une « réorganisation démocratique » de l'enseignement et se présente comme le projet d'un système unique d'éducation, idéalement ordonné à la promotion d'une classe dirigeante par son seul mérite - proprio sensu, une aristocratie - dans une société démocratique. Parmi les défaillances des structures d'enseignement de la Troisième République, É. Maneuvrier dénonce le « hiatus entre le premier et le second degré des écoles publiques ", qui interdit à un élève ayant achevé ses études dans le premier degré d'entrer " de plainpied dans celles du second » (p. 48). Les défenseurs de l'enseignement secondaire, en effet, s'accrochent à la conviction que les éléments premiers du savoir doivent être enseignés diversement, selon que l'on s'y limite, ou que l'on en fasse les prémisses d'un long curriculum désintéressé. Or, pour É. Maneuvrier, le principe démocratique est incompatible avec une organisation scolaire qui classe «les jeunes Français, dès leurs premiers pas dans la vie, en deux catégories irréductibles, ou peu s'en faut » (p. 49) et menace en permanence l'unité du corps social : "d'un côté, dans les bonnes écoles, les privilégiés; de l'autre, dans les médiocres, les déshérités. Ainsi, on prépare l'antagonisme des classes et on perpétue le règne de la discorde sociale » (Ibid.).

Prenant au mot la rhétorique ferryste de « fusion des classes ", il réclame donc, comme condition de l'« égalité devant la première instruction » (Ibid., 
p. 84), la suppression des classes élémentaires des lycées et la mise sur pied d'une école primaire prolongée jusqu'à quatorze ans, qui serait fréquentée par tous les milieux sociaux sans exception : " La condition même de la démocratie, c'est la fusion des classes dans la grande idée de la patrie et de la solidarité communes. Or, c'est à l'école primaire que cette grande idée doit commencer à germer, dans la camaraderie des études et des jeux, et c'est au régiment qu'elle doit s'épanouir, dans la fraternité des armes » (Ibid., p. 50). L'enseignement secondaire commencerait ensuite, de la quatorzième année à la dix-huitième année, pour ceux-là seuls que leurs aptitudes rendraient capables de le suivre avec succès, dans une filière classique ou professionnelle, où les classes seraient remplacées par des groupes de niveaux.

Pour la première fois peut-être, du fait des titres universitaires de son auteur, de son statut social et de l'idéal méritocratique dont il se prévaut, L'éducation de la bourgeoisie sous la République met à l'épreuve la doctrine républicaine des ordres, en dénonçant, au nom des valeurs mêmes qui la sous-tendent, son élitisme social déguisé. L'ouvrage a été immédiatement commenté par des acteurs éminents de l'administration pédagogique républicaine, eux aussi agrégés de philosophie, Ferdinand Buisson, Henri Marion, Gabriel Compayré. Mais ceux-ci apprécient diversement la hardiesse des propositions d'É. Maneuvrier: leurs commentaires trahissent en effet des positionnements qui, à l'intérieur du même paradigme méritocratique, combinent selon des proportions singulièrement différentes l'aspiration au traitement équitable des élèves, quelle que soit leur origine, et la peur d'une démocratisation qui fragiliserait les cadres sociaux normatifs.

\section{École unique de 6 à 14 ans ? Un idéal de notre temps}

L'ouvrage d'É. Maneuvrier est à peine sorti des presses, en décembre 1887, que Ferdinand Buisson, directeur de l'enseignement primaire au ministère de l'Instruction publique, en livre un commentaire à la Revue pédagogique (Buisson, 1887). Or, loin de réfuter É. Maneuvrier, il rappelle que les vues qu'il propose comme « une sorte d'idéal encore lointain » ne sont pas chimériques, puisqu'elles existent déjà dans les faits aux États-Unis et en Suisse. Là, les enfants de toutes conditions fréquentent les mêmes écoles primaires puis, quoique moins nombreux, les premières années de l'école secondaire. Leur présence «pêle-mêle » dans les classes, ajoute-til, est même à la source de l'« instinct de l'égalité » de ces peuples, que Buisson, toutefois, prend soin de distinguer des «théories égalitaires 》 des socialistes: "Ce que M. Maneuvrier ose faire entrevoir à la démocratie française comme une sorte d'idéal encore lointain, c'est le fameux free school system, que personne ne songe plus à défendre, à louer, à célébrer, attendu que c'est une cause définitivement gagnée depuis cinquante ans dans les anciens États de l'Union, depuis vingt-cing ans dans les autres. Et sans aller si loin, arrêtez-vous, lors de votre prochain voyage en Suisse, à Zurich, ou même à Genève, et vous aurez tout loisir de voir journellement réalisé le rêve de $M$. Maneuvrier: tous les matins vous verrez filles et garçons de sept à onze ans se rendre aux écoles primaires publiques, enfants des riches et enfants des pauvres, fils et filles de la bourgeoisie, voire de l'aristocratie, pêle-mêle avec ceux du petit artisan ou du petit boutiquier; puis vous les retrouverez encore, moins nombreux, mais aussi mêlés, à l'école secondaire de onze à quatorze ans en moyenne... Enfants, ils se sont toujours vus, toujours connus, toujours traités d'égal à égal, et plus tard, séparés par toutes les distances que la vie et la fortune mettront entre eux, ils se souviendront toujours de leurs jeunes années; et c'est pour cela que ce peuple a l'esprit démocratique, les mœurs démocratiques, l'instinct de l'égalité, qui n'est pas du tout la même chose que les théories égalitaires 》 (Buisson, 1887, p. 495) (5).

Surtout, poursuit F. Buisson, cette idée n'est pas seulement suisse ni américaine ; elle est une «idée française par excellence », que tous les auteurs de plans d'instruction publique sous la Révolution, par delà leurs divergences, ont partagée. Elle paraissait alors "l'évidence même ": la conception d'une instruction « indéfiniment graduée », par laquelle «on n'accède aux écoles secondaires qu'en sortant des écoles primaires » (Ibid., p. 496-497). Si l'audace révolutionnaire des termes d'《école primaire» et d'«école secondaire» est aujourd'hui effacée par l'usage, elle ressurgit dès qu'on les compare aux équivalents allemands couramment utilisés, Volksschule et Bürgerschule, école du peuple et école de la bourgeoisie : " deux classes d'établissements correspondant à deux classes sociales. C'est juste le contrepied de ce système, poursuit-il, qu'ont osé prendre les législateurs de 1792 » et, recueillant leur héritage, la Troisième République, qui a su retrouver «la définition primitive de l'enseignement primaire ». Certes, il partage le constat d'É. Maneuvrier, qu' « un abîme sépare encore l'ordre primaire des deux autres ». Les 
"barrières de la vanité ", les "préjugés d'un autre âge » concurrencent dans la bourgeoisie "l'intérêt de la fusion sociale » (Ibid., p. 499). Toutefois, rappelant les mots de Ferry, qui évoquait la "large issue " vers l'enseignement secondaire grâce aux bourses d'État, il souligne - c'était le moins que pût faire le Directeur de l'enseignement primaire - que grâce aux lois scolaires républicaines "un pont a été jeté sur cet abîme, passage étroit mais sûr, et qui pourra s'agrandir " (Ibid., p. 499).

\section{...une utopie funeste}

Cette appréciation bienveillante des thèses d'É. Maneuvrier n'a pas été du goût d'un autre cacique de la pédagogique républicaine, Gabriel Compayré (6). En janvier 1888, présidant l'assemblée générale de la Société pour l'étude des questions d'enseignement secondaire, qui défend les intérêts de cet ordre d'enseignement, il fait part dans son allocution d'ouverture de ses "expresses réserves » devant l'ouvrage. II y voit en effet «le plus violent réquisitoire " jamais écrit contre "notre système d'enseignement secondaire ", et s'en prend explicitement au commentaire favorable qu'en a livré par F. Buisson à la Revue pédagogique. (L'Université, journal des questions d'instruction publique, 1888 , p. 25). S'il concède que la revendication d'un enseignement commun, jusqu'à quatorze ans, aux fils de la bourgeoisie et à ceux du peuple, peut exercer un attrait sur " des âmes démocratiques, éprises d'égalité et de fraternité ", G. Compayré doute que ce "rêve" partagé par F. Buisson et É. Maneuvrier "puisse et doive jamais se réaliser». Reprochant particulièrement à Buisson de ne voir dans la séparation des ordres que "des bornes posées par la vanité, par l'esprit de caste", il déplore que les "raisons plus hautes, plus profondes" de ce clivage n'aient pas été avancées: à savoir, l'impossibilité de soumettre à un curriculum commun des enfants dont l'enseignement primaire, pour les uns, n'est qu'une " préface " à des études plus hautes, les studia humanitatis, enracinées dans l'apprentissage du grec et du latin, tandis qu'il est, pour les autres, une " journée sans lendemain ". "Les séduisantes rêveries humanitaires " de la fusion des classes sociales sur les bancs d'une même école sont donc sans poids devant "les nécessités pratiques des études ". Si l'on tentait pourtant de soumettre celles-ci à celles-là, on aggraverait encore l'«aberration de la pédagogie contemporaine" qu'a été, selon G. Compayré, la suppression par J. Ferry de l'étude des langues anciennes dans l'enseignement élé- mentaire des lycées, dans le but d'en rapprocher le curriculum de celui des classes de l'école primaire.

\section{...un idéal prématuré}

Quelques semaines plus tard, c'est au tour du philosophe Henri Marion, titulaire de la chaire de Science de l'éducation à la Sorbonne, de commenter longuement l'ouvrage d'É. Maneuvrier pour la Revue internationale de l'enseignement, organe de la Société de l'enseignement supérieur, dont il est l'un des membres du comité de rédaction (Marion, 1888). H. Marion connaît personnellement É. Maneuvrier, dont il a été le condisciple et l'ami à l'École normale supérieure (7). Détaillant les thèses de son ouvrage, il s'attarde, entre autres, sur celle que dénonçait G. Compayré, la transformation de l'enseignement primaire en "base uniforme et obligatoire " des autres degrés de l'instruction publique. H. Marion y accorde d'autant plus d'intérêt qu'il est lui-même l'un de ces sujets d'exception qui ont pu passer de l'école primaire au lycée. Contrairement à G. Compayré, il ne voit nulle "aberration " dans les propositions d'É. Maneuvrier. II augure qu'à l'avenir elles rencontreront de moins en moins de résistance dans la bourgeoisie, à mesure des progrès accomplis par l'enseignement primaire. Toutefois, il rejoint G. Compayré, en ne jugeant ni "libéral » ni nécessaire d'imposer actuellement à l'enfance bourgeoise cette promiscuité avec les élèves du primaire et préconise comme lui, pour ceux dont le curriculum élémentaire n'est qu'« une préparation à aller plus loin ", le maintien d'un enseignement "un peu différent ", dispensé "dans des maisons distinctes, par des maîtres différents ". II suffit, pour satisfaire l'exigence démocratique, que l'enseignement secondaire soit " constitué de manière à rester accessible à l'élite des écoles primaires ».

$\mathrm{H}$. Marion poursuit le même argumentaire dans son livre L'Éducation dans l'Université, publié en 1892, qui reproduit un cycle de conférences de pédagogie pratique données à la Sorbonne deux ans auparavant. Définissant l'enseignement primaire comme " premier degré de la culture, base nécessaire des deux autres ", il reprend la comparaison faite par F. Buisson avec le système scolaire allemand, pour partager avec lui l'idée que l'appellation de Volksschule - école du peuple - "répugne à nos mœurs démocratiques " et «répondrait mal chez nous à la réalité » (Marion, 1892, p. 40-42). La réalité française, telle que la perçoit $\mathrm{H}$. Marion, est celle d'une société dans laquelle il n'y a "plus de classes ", ou du 
moins, rectifie-t-il prudemment, «plus de barrières entre les classes ». II est dès lors légitime de projeter à l'horizon des réformes républicaines l'idée d'une école primaire commune, sur les bancs de laquelle les petits Français, délivrés de toute «prédestination » sociale, pourraient commencer «l'apprentissage de l'égalité » et, pour quelques-uns d'entre eux au moins, passer «de plain-pied» de l'école primaire à l'enseignement secondaire. Toutefois, I'horizon des réformes démocratiques une fois campé, $\mathrm{H}$. Marion s'empresse d'opposer des objections à la revendication trop hâtive de leur mise en œuvre. Elle pourrait être en effet très dommageable à l'enseignement public, si elle conduisait à négliger les attentes différenciées des clientèles sociales et celle de la nation elle-même, qui imposent notamment d'outiller l'enfance populaire d'une culture « appropriée à [sa] destinée probable»: "pour cet avantage, si grand qu'il soit, il ne faudrait pas fausser toute la notion de l'enseignement public, oublier les besoins divers des diverses catégories de citoyens, les services très distincts que le pays attend de ses enfants et qui demandent une préparation très différente. L'enseignement primaire doit donc bien répondre avant tout aux besoins les plus généraux, fournir aux enfants des travailleurs une culture appropriée à leur destinée probable, et qui forme un tout par elle-même " (lbid.).

\section{**}

La critique par É. Maneuvrier, à partir des valeurs républicaines elles-mêmes, de l'organisation scolaire des années 1880 et ses propositions d'une école unique de six à quatorze ans mettent ainsi à jour, par les réactions qu'elles ont suscitées, le caractère composite du compromis scolaire républicain. Elles ont en effet conduit trois figures de premier plan de la pédagogie républicaine à la formulation d'autant de variantes du même paradigme méritocratique : si F. Buisson paraît prêt, en son nom, dès la fin de la décennie 1880, à œuvrer pour la mixité sociale des premières classes, G. Compayré refuse qu'il serve de caution à la moindre remise en cause de la spécificité de l'ordre secondaire classique; quant à $\mathrm{H}$. Marion, favorable dans l'idéal à l'école primaire pour tous, il juge pourtant prudent de ne pas heurter les aspirations ségrégatives de la clientèle bourgeoise; repoussant à plus tard toute tentative de réforme, il rejoint, de facto, le conservatisme social de G. Compayré.

\section{CONCLUSION}

La politique scolaire républicaine des années fondatrices de la Troisième République s'est construite dans la tension entre un héritage révolutionnaire revendiqué - égalité de tous devant l'instruction, gratuité, laïcité - et la poursuite d'une politique de "modernisation » des structures, initiée par les élites éclairées des régimes précédents et ordonnée à l'ajustement de l'offre scolaire aux besoins émergeants et différenciés du social. Dans le débat public de la décennie 1880 , cette tension est au principe de prises de positions variées, ouvrant un spectre opposant, aux bornes extrêmes, l'idée socialiste - et communarde - d'éducation intégrale et, à l'opposé, le refus de toute réforme de l'ordre secondaire classique qui compromettrait la continuité de son curriculum linéaire, la pureté de ses contenus désintéressés et la qualité de son public. Entre ces pôles, en filigrane sous la variété des positions exprimées, deux modèles scolaires distincts sont repérables, se revendiquant du même idéal d'un progrès démocratique. Le premier, qui sous-tend la politique de J. Ferry, se réfère au principe d'un enseignement universel des connaissances premières, mais en des lieux séparés, conformément à la diversité des clientèles sociales. Son caractère ségrégatif évident, en dépit de la finalité " libérale » octroyée par J. Ferry à l'enseignement primaire, est amorti par la possibilité d'un accès au second degré offert aux meilleurs éléments de l'école populaire, grâce aux bourses, dont les esprits avancés escomptent l'accroissement progressif. Le second modèle, auquel Ferdinand Buisson lui-même paraît adhérer vers 1887 , au moins mezzo voce, reconduit plus directement que J. Ferry au projet condorcetien. II revendique la constitution d'une école primaire universelle rassemblant pour un temps la progéniture de toutes les clientèles sociales et un passage " de plain-pied " à des études prolongées pour une partie d'entre elle, au vu des seules aptitudes. Largement minoritaire dans les années 1880 , il préparait pourtant plus que l'autre à l'idée d'un dépassement d'une organisation scolaire dont les différenciations commençaient à apparaître trop visiblement déterminées par les différences de loisir et de condition (8).

Patrick Dubois

patrick.dubois@dijon.iufm.fr IUFM de Bourgogne

UMR Éducation \& Politiques

(INRP-université Lumière-Lyon 2) 
(1) Archives Nationales, F/17/22163, dossier personnel « Foncin ».

(2) Briand \& Chapoulie, 1992, p. 372. Selon un observateur du temps, si " on répugne invinciblement en France " à exhiber les distinctions sociales, au contraire de l'Allemagne, que l'expression d'“ écoles bourgeoises " n'effraie pas, leur légitimité n'est généralement pas remise en cause : "nous subissons bien des classes de fait, parce qu'elles s'imposent, mais nous ne voulons pas qu'elles soient comme affichées » (Marguerin, 1878, p. 354).

(3) Créé sous le Second Empire par Victor Duruy (circulaire du 2 octobre 1863, loi du 21 juin 1865), l'« enseignement secondair spécial ", prolongeait et amplifiait des tentatives d'enseignement intermédiaire, entre l'école populaire et le secondaire classique, engagées dès la fin de la Restauration (Prost, 1968).

(4) Dès 1881 , la création d'un corps de professeurs des classes élémentaires des lycées, recruté par concours spécial, entérine la distinction des corps professionnels primaire et secondaire de l'enseignement élémentaire (Robert, 2005).

(5) Le « rêve " d'É. Maneuvrier n'est toutefois « journellement réalisé " en Suisse qu'au prix de quelques simplifications que Buisson s'autorise. Ainsi, à Genève, si depuis la loi de 1886 tous les enfants de six à douze ans se côtoient en théorie sur les mêmes bancs, les écoles privées et la ségrégation urbaine limitent cette mixité sociale ; en outre, dès 11 ans, les élèves se destinant à de longues études reçoivent une formation préparatoire séparée (Hofstetter, 1998, p. 322).

(6) Docteur ès lettres, ancien professeur à la Faculté des lettres de Toulouse, puis chargé de cours de pédagogie à l'École normale supérieure de jeunes filles de Fontenay, il est depuis 1881 député républicain.

(7) Bulletin de l'ENS, 1918, p. 43-47.

(8) II conduira F. Buisson, déposant en 1899 devant la Commission parlementaire sur l'enseignement secondaire présidée par Alexandre Ribot, à revendiquer, comme une "exigence de la démocratie ", un élargissement de l'assiette sociale de l'enseignement secondaire, et corrélativement, à la transformation du curriculum des lycées au profit d'un plan d'études " mixte " associant à une "large culture libérale », un " solide enseignement, ne disons pas utilitaire, mais utile, assurant à l'élève la certitude de gagner sa vie " (Enquête sur l'enseignement secondaire. Journal officiel. Documents parlementaires. Annexe au procès verbal de la $2^{\mathrm{e}}$ séance du 28 mars 1899 , t. 1, p. 435-444).

\section{BIBLIOGRAPHIE}

Beaussire É. (1879). "Le projet de loi sur l'enseignement supérieur ", Revue politique et littéraire, 15 février, p. 765-771.

BoIssien G. (1880). «Le nouveau plan d'études », Revue des deux mondes, $1^{\mathrm{er}}$ septembre, p. 101-123.

Briand J.-P. \& Chapoulie J.-M. (1992). Les collèges du peuple: l'enseignement primaire supérieur et le développement de la scolarisation prolongée sous la Troisième République. Paris : INRP ; CNRS ; Fontenayaux-Roses : ENS de Fontenay - Saint-Cloud.

BuIsson F. (1878). Rapport sur l'instruction primaire à l'Exposition universelle de Philadelphie en 1876. Paris : Imprimerie nationale.

Buisson F. (1878-1887). Dictionnaire de pédagogie et d'instruction primaire. Paris : Hachette, 4 t. (1 $1^{\text {re éd.) }}$

Buisson F. (1887). "L'éducation de la bourgeoisie sous la République ", Revue pédagogique, $2^{\mathrm{e}}$ semestre, p. 491-507.

Chapoulie J.-M. (1989). "L'enseignement primaire supérieur de la loi Guizot aux écoles de la III République ». Revue d'histoire moderne et contemporaine, vol. 36, $n^{\circ} 3$, p. 413-437.

Demeulenaere-Douyère C. (1994). Paul Robin (1837-1912): un militant de la liberté et du bonheur. Paris : Publisud.

FERRY Jules (1881). "Rapport au Président de la République, 29 octobre 1881 ", Revue pédagogique, $2^{\mathrm{e}}$ semestre, p. 675-678.

FerRY J. (1893-1898). Discours et opinions de Jules Ferry, publiés avec commentaires et notes, par Paul Robiquet. Paris : A. Colin, 7 vol.

GOBLET R. (1885). "Discours prononcé à la distribution des prix aux élèves de l'Association philotechnique ", Manuel général, p. 331-333.

GrÉARD O. (1887). Éducation et instruction. Paris : Hachette, $4 \mathrm{vol}$.
JoLY H. (1885). "L'éducation intégrale. La démocratie et les études ", La nouvelle revue. 15 juillet, p. 277-311.

HOFSTETTER R. (1998). Les lumières de la démocratie: histoire de l'école primaire publique à Genève au XIXe siècle. Bern : P. Lang.

MANeuvrier É. (1888). L'Éducation de la bourgeoisie sous la République. Paris : Éd. du Cerf.

MARguerin É. (1878). «À propos du projet de loi en préparation sur l'enseignement primaire supérieur ", Revue pédagogique, $1^{\mathrm{er}}$ semestre, p. 345-359.

MARGUERIN É. (1881). "Mémoire de M. le Vice-Recteur sur l'enseignement secondaire spécial », Revue pédagogique, $1^{\mathrm{er}}$ semestre, p. 121-135.

MARION H. (1888). "L'éducation de la bourgeoisie sous la République ", Revue internationale de l'enseignement, t. $15,1^{\text {er }}$ semestre, p. $132-152$.

MARION H. (1892). L'éducation dans I'Université. Paris: A. Colin.

PÉCAUT F. (1879). Études au jour le jour sur l'éducation nationale. Paris : Hachette.

Prost A. (1968). Histoire de l'enseignement en France 18001967. Paris : A. Colin.

Robert A. D. (2005). « Les professeurs des classes élémentaires des lycées et leur représentation: crépuscule et postérité d'une idéologie catégorielle (1881-1965)». In P. Caspard, J.-N. Luc \& P. Savoie (dir.), Lycées, lycéens, lycéennes: deux siècles d'histoire. Lyon: INRP, p. 317-329.

Rosanvallon P. (1985). Le moment Guizot. Paris : Gallimard.

SIMON J. (1888). "La pédagogie de M. Gréard». Revue pédagogique, $1^{\mathrm{er}}$ semestre, p. 193-197.

THIVEND M. (2006). L'école républicaine en ville : Lyon, 18701914. Paris : Belin. 